HIV

\title{
Epidemiology of HIV among black and minority ethnic men who have sex with men in England and Wales
}

\author{
S Dougan, J Elford, B Rice, A E Brown, K Sinka, B G Evans, O N Gill, K A Fenton
}

Sex Transm Infect 2005;81:345-350. doi: 10.1136/sti.2004.012328

See end of article for authors' affiliations

Correspondence to: Sarah Dougan,

Department of HIV and Sexually Transmitted Infections, Communicable Disease Surveillance Centre, Health Protection Agency, Centre for Infections, 61 Colindale Avenue, London, NW9 5EQ, UK; sarah.dougan@ hpa.org.uk

Accepted for publication 28 November 2004

\begin{abstract}
Objectives: To examine the epidemiology of HIV among black and minority ethnic (BME) men who have sex with men (MSM) in England and Wales (E\&W).

Methods: Ethnicity data from two national HIV/AIDS surveillance systems were reviewed (1997-2002 inclusive), providing information on new HIV diagnoses and those accessing NHS HIV treatment and care services. In addition, undiagnosed HIV prevalence among MSM attending 14 genitourinary medicine (GUM) clinics participating in the Unlinked Anonymous Prevalence Monitoring Programme and having routine syphilis serology was examined by world region of birth.

Results: Between 1997 and 2002, 1040 BME MSM were newly diagnosed with HIV in E\&W, representing $12 \%$ of all new diagnoses reported among MSM. Of the 1040 BME MSM, 27\% were black Caribbean, $12 \%$ black African, 10\% black other, $8 \%$ Indian/Pakistani/Bangladeshi, and $44 \%$ other/mixed. Where reported $(n=395), 58 \%$ of BME MSM were probably infected in the United Kingdom. An estimated 7.4\% (approximate $95 \% \mathrm{Cl}: 4.4 \%$ to $12.5 \%$ ) of BME MSM aged 16-44 in E\&W were living with diagnosed HIV in 2002 compared with $3.2 \%$ (approximate $95 \% \mathrm{Cl}: 2.6 \%$ to $3.9 \%$ ) of white MSM ( $<<0.001$ ). Of Caribbean born MSM attending GUM clinics between 1997 and 2002, the proportion with undiagnosed HIV infection was $15.8 \%$ (95\% Cl: $11.7 \%$ to $20.8 \%$ ), while among MSM born in other regions it remained below 6.0\%.

Conclusions: Between 1997-2002, BME MSM accounted for just over one in 10 new HIV diagnoses among MSM in E\&W; more than half probably acquired their infection in the United Kingdom. In 2002, the proportion of BME MSM living with diagnosed HIV in E\&W was significantly higher than white MSM. Undiagnosed HIV prevalence in Caribbean born MSM was high. These data confirm the need to remain alert to the sexual health needs and evolving epidemiology of HIV among BME MSM in E\&W.
\end{abstract}

M en who have sex with men (MSM) remain the group at greatest risk of acquiring HIV in England and Wales $(\mathrm{E} \& W) .{ }^{1}$ Recent years have seen a substantial rise in the number of new HIV diagnoses among these men. While the ethnicity of people with heterosexually acquired HIV has been examined in detail, ${ }^{12}$ there has been little consideration of HIV among black and minority ethnic (BME) MSM in E\&W.

An estimated 10 000-30 000 BME MSM aged 16-44 years currently live in E\&W, according to population prevalence estimates from the National Survey of Sexual Attitudes and Lifestyles (Natsal 2000) applied to the 2001 census, $^{3-5}$ (Dr Catherine Mercer, personal communication). Black communities in E\&W, particularly black Caribbean and African communities, experience poor sexual health with relatively high rates of bacterial STIs. ${ }^{67}$ There is also evidence that BME MSM in E\&W are more likely to report high risk sexual behaviour than other MSM while lacking culturally appropriate information, safe spaces, and social networks to meet their sexual health needs. ${ }^{8}$ In the United States, BME MSM bear a disproportionate burden of incident HIV; among young BME MSM surveyed in six US cities between 1994 and 2000, HIV incidence was $14.7 \%$ compared to $2.5 \%$ among white MSM. ${ }^{9}$ To avert a similar public health crisis in E\&W, HIV infection among BME MSM must be monitored and appropriate action taken.

This paper examines the epidemiology of HIV among BME MSM in E\&W to provide an evidence base for clinicians, policy makers and those involved in health promotion. In this paper we focus on new HIV diagnoses, those accessing HIV treatment and care services within the National Health Service (NHS), and the prevalence of undiagnosed HIV.

\section{METHODS}

Data for 1997-2002 from three national HIV surveillance systems held at the Communicable Disease Surveillance Centre (CDSC) were examined. Analyses were limited to E\&W to ensure comparability between sources.

\section{New HIV diagnoses}

CDSC receives voluntary confidential reports of new HIV diagnoses from laboratories (since 1985) and clinicians (since 2000). ${ }^{10}$ Data on probable route of infection (for example, sex between men) are collected on all reports, and followed up by a research nurse where incomplete. Information on ethnicity and probable country of infection is also collected. Country of birth has been included in clinician reports of HIV diagnoses since 2000. A "late" diagnosis is defined as an AIDS diagnosis within 3 months of an HIV diagnosis.

\section{Survey of Prevalent HIV Infections Diagnosed (SOPHID)}

SOPHID has provided an estimate of the number of individuals living with diagnosed HIV infection in E\&W since 1995. ${ }^{11}$ The survey aims to include every individual living in E\&W with diagnosed HIV infection who has attended for HIV treatment or care at NHS services during

Abbreviations: $A R T$, antiretroviral therapy; $B M E$, black and minority ethnic; CDSC, Communicable Disease Surveillance Centre; E\&W, England and Wales; GUM, genitourinary medicine; IPB, Indian/ Pakistani/Bangladeshi; IQR, interquartile ranges; MSM, men who have sex with men; NHS, National Health Service; SOPHID, Survey of Prevalent HIV Infections Diagnosed; UAPMP, Unlinked Anonymous Prevalence Monitoring Programme; VCT, voluntary confidential testing 
a calendar year. Data collected include ethnicity, probable route of infection, level of antiretroviral therapy (ART), CD4 count when last seen, and area of residence.

\section{Unlinked Anonymous Prevalence Monitoring Programme (UAPMP)}

The UAPMP has measured the prevalence of undiagnosed HIV infection in different population subgroups since $1990 .{ }^{12}$ The genitourinary medicine (GUM) clinic survey uses residual blood taken for syphilis serology, providing information on HIV prevalence among MSM attending GUM clinics. A GUM clinic is synonymous with an STI clinic. Clinics provide treatment and care for patients with STIs and HIV. These free and strictly confidential services are open access; patients can refer themselves without going through their primary care physician. Fourteen (of 228) GUM clinics in E\&W participate in the UAPMP GUM survey (seven (of 34) in London, seven (of 194) elsewhere in $\mathrm{E} \& W$ ). Information is collected on co-infection with acute STIs and the uptake of voluntary confidential testing (VCT). The term "previously undiagnosed HIV" only includes samples from HIV infected MSM who had not had a positive voluntary HIV test before clinic attendance; they were either newly diagnosed during the clinic visit or left without accepting VCT, therefore remaining undiagnosed. In the UAPMP all samples have patient identifying details irreversibly removed before testing for HIV infection.

\section{Ethnicity}

Ethnicity is established during clinic visits with the patient. Surveillance forms are subsequently completed by a healthcare worker, typically using patient notes. There is no reclassification at CDSC unless there is an obvious error in form completion. Broad ethnicity categories are used, collapsed from the 1991 census so that population denominators can be applied. These categories did not change over the period 1997-2002 and are white, black African, black Caribbean, black other, Indian/Pakistani/Bangladeshi (IPB), and other/mixed. "Black other" includes "black British" while "other/mixed" includes men from South America and Asia (excluding IPB). Those of black and white ancestry will be classified as "black other" or "other/mixed," depending on assignment at the clinic level. BME includes all these categories, except white.

Ethnicity data have been collected on laboratory reports of HIV diagnoses since 1996, on clinician reports of HIV/AIDS diagnoses since 2000 and were collected on AIDS reports until 2000. Completeness of ethnicity data on reports of MSM newly diagnosed with HIV ranged from $58 \%$ to $81 \%$ between 1997 and 2002. Ethnicity data have also been collected in the SOPHID surveys since they began in 1995; completeness ranged from $96 \%$ to $98 \%$ between 1997 and 2002. To improve the completeness of ethnicity data for new HIV diagnoses, reports among MSM where ethnicity was missing were matched to the SOPHID database using soundex code, sex, date of birth and other available information. If ethnicity was recorded in SOPHID it was used to classify the ethnicity of MSM newly diagnosed with HIV. The UAPMP GUM survey does not collect information on ethnicity. Region of birth is collected instead.

For these analyses, countries of birth or infection outside the United Kingdom were aggregated into broader regions. Men who were born or probably infected in England, Wales, Northern Ireland, or Scotland were classified as being born or infected in the United Kingdom. This classification could not be disaggregated.

\section{Statistical methods}

Where appropriate, data were analysed using Epi-Info 6 (v.6.04d) and Stata 8, with categorical variables analysed using $\chi^{2}$ tests. Interquartile ranges (IQR) for medians are presented. Data for E\&W were analysed for 1997-2002. Those without reported ethnicity (new diagnoses, SOPHID) or country of birth (UAPMP) were excluded. New diagnoses are based on reports received by the end of December 2003. Statistical inferences were only made for data from sample populations (UAPMP GUM survey and estimates of the proportions of BME and white MSM living with diagnosed HIV), and not surveillance data relating to whole populations (new diagnoses and SOPHID).

The proportion of BME and white MSM living with diagnosed HIV in E\&W was calculated by dividing the number of BME or white MSM aged 16-44 accessing HIV services (SOPHID) by the number of BME or white MSM in $\mathrm{E} \& \mathrm{~W}$. The denominators were calculated by multiplying the point estimate for the proportion of BME or white MSM aged 16-44 residing in E\&W (from Natsal 2000) with the number of BME or white men aged 16-44 enumerated in the 2001 census, $^{3-5}$ (Dr Catherine Mercer, personal communication). Applying the $95 \%$ confidence intervals around the Natsal point estimates to the census data allowed us to determine approximate $95 \%$ CIs for the proportion of men living with diagnosed HIV.

\section{Ethics}

Reports of new diagnoses and of those diagnosed and living with HIV are voluntary and confidential. To maintain patient confidentiality no names are held on the database, soundex codes are used instead. ${ }^{13}$ The reporting system has approval under the section 60 regulations of the Health and Social Care Act (Statutory Instrument 1438-June 2002). The ethical and legal basis for UAPMP has been described elsewhere. ${ }^{14}$ In short, best practice guidance states that for purposes of public health surveillance, and where samples are anonymised, specific patient consent is not required, on condition that "active local arrangements" allow patients the opportunity to be aware of what may happen to their samples and mechanisms are in place for respecting spontaneous objections. The programme complies with guidelines published by the Medical Research Council, ${ }^{15}$ and Department of Health interim guidelines on the use of human organs and tissue. ${ }^{16}$ All data are stored on restricted and secure databases at CDSC, with strict adherence to the Data Protection Act and Caldicott Guidelines. ${ }^{17}$

\section{RESULTS}

\section{New diagnoses}

Between 1997 and 2002, 1040 BME MSM were newly diagnosed with HIV, representing 12\% (1040/8861) of all new diagnoses reported among MSM in E\&W in that period (table 1). The number of new diagnoses among both BME and white MSM increased between 1997-2002. The contribution of BME MSM to the total number of new HIV diagnoses among all MSM did not vary significantly from one year to the next. Of the BME MSM newly diagnosed with HIV between 1997-2002 (1040), 27\% (276) were black Caribbean, 12\% (124) black African, 10\% (101) black other, $8 \%$ (85) IPB, and 44\% (454) other/mixed. The percentage of newly diagnosed BME MSM who were black Caribbean or black African increased during the study period. Median age at diagnosis was 32 years (IQR: 28-37) for BME MSM compared to 35 years (IQR: 29-4l) for white MSM.

Overall, 11\% (118/1040) of BME MSM were diagnosed "late" compared with 13\% $(927 / 7,062)$ of white MSM. There was some evidence that the proportion of late diagnoses differed between BME groups: 19\% (16/85) of IPB MSM were diagnosed late, $14 \%(38 / 276)$ of black Caribbeans, 10\% (10/ $101)$ of black other, $10 \%(44 / 454)$ of other/mixed, and $8 \%$ (10/124) of black Africans. 
Table 1 New HIV diagnoses among MSM in England and Wales by ethnicity and year of diagnosis, 1997-2002

\begin{tabular}{|c|c|c|c|c|c|c|c|c|c|c|c|c|c|c|}
\hline \multirow[b]{3}{*}{ Ethnic group } & \multicolumn{14}{|c|}{ Year of diagnosis } \\
\hline & \multicolumn{2}{|l|}{1997} & \multicolumn{2}{|l|}{1998} & \multicolumn{2}{|l|}{1999} & \multicolumn{2}{|l|}{2000} & \multicolumn{2}{|l|}{2001} & \multicolumn{2}{|l|}{2002} & \multicolumn{2}{|l|}{ Total } \\
\hline & No & $\%$ & No & $\%$ & No & $\%$ & No & $\%$ & No & $\%$ & No & $\%$ & No & $\%$ \\
\hline Black and minority ethnic & 164 & 12.3 & 137 & 10.7 & 136 & 10.5 & 178 & 12.5 & 218 & 13.0 & 207 & 12.4 & 1040 & 12.0 \\
\hline White & 1073 & 80.8 & 1084 & 84.6 & 1078 & 83.2 & 1154 & 80.8 & 1359 & 80.9 & 1314 & 78.8 & 7062 & 81.4 \\
\hline Not reported & 91 & 6.9 & 61 & 4.8 & 81 & 6.3 & 97 & 6.8 & 102 & 6.1 & 147 & 8.8 & 579 & 6.7 \\
\hline All MSM & 1328 & 100.0 & 1282 & 100.0 & 1295 & 100.0 & 1429 & 100.0 & 1679 & 100.0 & 1668 & 100.0 & 8681 & 100.0 \\
\hline Black African & 8 & 4.9 & 15 & 10.9 & 13 & 9.6 & 28 & 15.7 & 27 & 12.4 & 33 & 15.9 & 124 & 11.9 \\
\hline Black Caribbean & 33 & 20.1 & 32 & 23.4 & 42 & 30.9 & 42 & 23.6 & 61 & 28.0 & 66 & 31.9 & 276 & 26.5 \\
\hline Black other & 21 & 12.8 & 15 & 10.9 & 15 & 11.0 & 14 & 7.9 & 18 & 8.3 & 18 & 8.7 & 101 & 9.7 \\
\hline IPB & 8 & 4.9 & 13 & 9.5 & 12 & 8.8 & 19 & 10.7 & 23 & 10.6 & 10 & 4.8 & 85 & 8.2 \\
\hline Other/Mixed & 94 & 57.3 & 62 & 45.3 & 54 & 39.7 & 75 & 42.1 & 89 & 40.8 & 80 & 38.6 & 454 & 43.7 \\
\hline All BME MSM & 164 & 100.0 & 137 & 100.0 & 136 & 100.0 & 178 & 100.0 & 218 & 100.0 & 207 & 100.0 & 1040 & 100.0 \\
\hline
\end{tabular}

Probable country of infection was reported for 38\% (395/ 1040) of BME MSM. Of these men, 58\% (228/395) were probably infected in the United Kingdom. The percentage of BME MSM probably infected with HIV in the United Kingdom varied by ethnic group: black Africans 39\% (22/56); other/ mixed 55\% (90/165); black Caribbean 61\% (62/102); black other $70 \%$ (26/37); and IPB, 80\% (28/35). Of the $603 \mathrm{BME}$ MSM diagnosed between 2000 and 2002, 53\% (321/603) had country of birth reported. Of these, $29 \%$ (93/321) were born in the United Kingdom. This varied by ethnic group: black Africans 16\% (7/45); other/mixed 19\% (20/107); black Caribbean 35\% (35/100); IPB 42\% (15/36); and black other $48 \%(16 / 33)$. Nearly a third (34/107) of other/mixed ethnicity MSM were born in Central/South America, with a further third (34/107) born in Asia (excluding India/Pakistan/Bangladesh) and nearly 20\% (20/107) in the United Kingdom.

Both probable country of infection and country of birth were reported for 197 BME MSM diagnosed between 2000 and 2002 . Overall $60 \%$ (119/197) were probably infected in the United Kingdom while 30\% (60/197) were born here. Of 32 black African MSM, 38\% (12/32) were born in Africa but probably infected in the United Kingdom while 50\% (16/32) were born and probably infected in Africa. For black Caribbean MSM (60), 33\% (20/60) were born and probably infected in the United Kingdom, 27\% (16/60) were born in the Caribbean but probably infected in the United Kingdom, while $37 \%(22 / 60)$ were born and probably infected in the Caribbean.

\section{Prevalent diagnosed infections}

In 1997, 10\% $(921 / 9,117)$ of all MSM receiving HIV treatment or care reported to the SOPHID survey were BME, rising to $12 \%(1625 / 13$ 990) in 2002 (table 2). Between 1997-2002, among BME MSM receiving HIV treatment or care, the proportion who were black African, black Caribbean, or IPB increased. Based on data from Natsal and the 2001 census we estimated that $7.4 \%$ (approximate $95 \%$ CI: $4.4 \%$ to $12.5 \%$ ) of all BME MSM aged 16-44 in E\&W were living with diagnosed HIV in 2002 compared with 3.2\% (approximate 95\% CI: $2.6 \%$ to $3.9 \%$ ) of white MSM aged $16-44$ ( $p<0.001$ ).

While the majority of BME MSM receiving treatment and care lived in London, this decreased from $88 \%$ (810/921) in 1997 to $83 \%$ (1345/1625) in 2002. CD4 count and level of ART at last clinic attendance were reported for $88 \%$ (1433/1625) of BME MSM and 88\% ( 10 480/1 1 954) of white MSM in 2002. Seventy one per cent (130/182) of BME MSM with a CD4 cell count $<200$ cells $\times 10^{6} / 1$ for whom level of ART was known, were receiving triple therapy or more, compared to $77 \%$ (1045/1353) of white MSM. The proportion recorded as taking ART at last clinic attendance was equivalent among BME groups.

\section{Undiagnosed prevalence}

Between 1997 and 2002, 4.3\% (95\% CI: 4.1\% to 4.5\%) of all MSM attending GUM clinics in the UAPMP survey were HIV infected and had not been previously diagnosed, ranging from $3.7 \%$ (95\% CI: $2.4 \%$ to $5.3 \%$ ) among Asian born men to

Table 2 MSM resident in England and Wales and seen for treatment and care at NHS services by ethnicity and year of SOPHID survey, 1997-2002

\begin{tabular}{|c|c|c|c|c|c|c|c|c|c|c|c|c|}
\hline & \multicolumn{12}{|c|}{ Year seen for HIV related treatment or care } \\
\hline & \multicolumn{2}{|l|}{1997} & \multicolumn{2}{|l|}{1998} & \multicolumn{2}{|l|}{1999} & \multicolumn{2}{|l|}{2000} & \multicolumn{2}{|l|}{2001} & \multicolumn{2}{|l|}{2002} \\
\hline & No & $\%$ & No & $\%$ & No & $\%$ & No & $\%$ & No & $\%$ & No & $\%$ \\
\hline Black and minority ethnic & 921 & 10.1 & 1056 & 10.7 & 1169 & 10.9 & 1260 & 10.9 & 1414 & 11.1 & 1625 & 11.6 \\
\hline White & 7869 & 86.3 & 8546 & 86.7 & 9331 & 86.6 & 9985 & 86.4 & 10921 & 86.0 & 11954 & 85.4 \\
\hline Not reported & 327 & 3.6 & & 2.6 & 273 & 2.5 & 317 & 2.7 & 359 & 2.8 & 411 & 2.9 \\
\hline All MSM & 9117 & 100.0 & 9862 & 100.0 & 10773 & 100.0 & 11562 & 100.0 & 12694 & 100.0 & 13990 & 100.0 \\
\hline Black African & 83 & 9.0 & 97 & 9.2 & 125 & 10.7 & 139 & 11.0 & 154 & 10.9 & 197 & 12.1 \\
\hline Black Caribbean & 147 & 16.0 & 159 & 15.1 & 188 & 16.1 & 214 & 17.0 & 266 & 18.8 & 299 & 18.4 \\
\hline Black other & 135 & 14.7 & 145 & 13.7 & 142 & 12.1 & 147 & 11.7 & 165 & 11.7 & 192 & 11.8 \\
\hline IPB & 42 & 4.6 & 54 & 5.1 & 62 & 5.3 & 69 & 5.5 & 80 & 5.7 & 92 & 5.7 \\
\hline Other/mixed & 514 & 55.8 & 601 & 56.9 & 652 & 55.8 & 691 & 54.8 & 749 & 53.0 & 845 & 52.0 \\
\hline All BME MSM & 921 & 100.0 & 1056 & 100.0 & 1169 & 100.0 & 1260 & 100.0 & 1414 & 100.0 & 1625 & 100.0 \\
\hline
\end{tabular}


Table 3 Prevalence of previously undiagnosed $\mathrm{HIV}^{*}$, proportion remaining undiagnosed after clinic visit and uptake of voluntary confidential testing among MSM attending 14 GUM clinics in England \& Wales by region or country of birth, 19972002

\begin{tabular}{|c|c|c|c|c|c|c|c|}
\hline & \multicolumn{3}{|c|}{ Undiagnosed prevalence* } & \multirow{2}{*}{\multicolumn{2}{|c|}{$\begin{array}{l}\text { Proportion remaining undiagnosed after } \\
\text { clinic visit } \\
\text { (No of HIV positive samples from } \\
\text { individuals who could have been } \\
\text { diagnosed at clinic visit but left } \\
\text { clinic unaware of infection/ } \\
\text { no of HIV positive samples*) }\end{array}$}} & \multicolumn{2}{|c|}{ Proportion accepting VCT } \\
\hline & \multicolumn{3}{|c|}{$\begin{array}{l}\text { (No of HIV positive samples* / } \\
\text { no of samples tested*) }\end{array}$} & & & \multicolumn{2}{|c|}{$\begin{array}{l}\text { (No of samples from individuals } \\
\text { accepting VCT/no of samples } \\
\text { tested*) }\end{array}$} \\
\hline & $\%$ & $95 \% \mathrm{Cl}$ & $\mathrm{n} / \mathrm{N}$ & $\%$ & $n / N$ & $\%$ & $n / N$ \\
\hline Caribbean & 15.8 & (11.7 to 20.8$)$ & $42 / 265$ & 45.2 & $19 / 42$ & 52.1 & $138 / 265$ \\
\hline Central/South America & 5.7 & (4.4 to 7.4$)$ & $57 / 993$ & 57.9 & $33 / 57$ & 59.7 & $593 / 993$ \\
\hline Sub-Saharan Africa & 4.6 & (3.3 to 6.2 ) & $40 / 870$ & 62.5 & & 54.4 & $473 / 870$ \\
\hline Asia & 3.7 & (2.4 to 5.3$)$ & $27 / 739$ & 51.9 & $14 / 27$ & 56.4 & $417 / 739$ \\
\hline United Kingdom & 4.0 & (3.7 to 4.2 ) & $971 / 24452$ & 60.2 & $585 / 971$ & 51.9 & $12691 / 24452$ \\
\hline Totalt & 4.3 & (4.1 to 4.5 ) & $1595 / 37039$ & 60.2 & $960 / 1595$ & 52.3 & $19387 / 37039$ \\
\hline \multicolumn{8}{|c|}{$\begin{array}{l}\text { MSM, men who have sex with men. } \\
\text { *MSM with "previously undiagnosed HIV" includes samples from HIV infected MSM whose HIV infection was not diagnosed before clinic attendance, but may } \\
\text { either have had a new diagnosis at the clinic visit or left remaining undiagnosed. It excludes men with a previously diagnosed HIV infection. } \\
\text { †Total includes men born in Australia and Oceania, Middle Eastern Crescent, North America, rest of Europe, and those where region of birth was not recorded. }\end{array}$} \\
\hline
\end{tabular}

$15.8 \%$ (95\% CI: $11.7 \%$ to $20.8 \%$ ) among Caribbean born men $(\mathrm{p}<0.001)$ (table 3$)$. The percentage of Caribbean born MSM attending GUM clinics with previously undiagnosed HIV significantly increased over the period (from $5.4 \%$ (95\% CI: $0.6 \%$ to $18.2 \%$ ) to $31.4 \%$ (95\% CI: $20.9 \%$ to $43.6 \%$ ), p $<0.001$ ). However, data for 2003 show a decrease in the prevalence of undiagnosed HIV among Caribbean born MSM (7.5\% (3/ $40)$ ). With 2003 data included, the test for trend was only of borderline significance $(p=0.06)$. For MSM born in Asia, there was some evidence that the proportion with previously undiagnosed HIV decreased over time (from 5.5\% (95\% CI: $2.0 \%$ to $11.6 \%$ ) to $1.2 \%$ (95\% CI: $1.5 \%$ to $4.3 \%$ ), $\mathrm{p}=0.07$ ), while for men born in Central/South America, sub-Saharan Africa, and the United Kingdom there were no significant changes over time $(\mathrm{p}=0.3)$.

Overall, 60\% (960/1595) of HIV infected MSM whose HIV infection could have been diagnosed remained unaware of their infection after leaving the clinic (table 3). This did not vary significantly by region of birth (including the United Kingdom $)(p=0.4)$.

Among MSM attending GUM clinics with previously undiagnosed HIV, 52\% $(19,387 / 37,039)$ accepted VCT. This varied significantly by region of birth $(\mathrm{p}<0.001)$, but all were in the range of $52 \%$ to $60 \%$ (table 3). Between 1997-2002, uptake of VCT significantly increased among MSM regardless of region of birth $(\mathrm{p}<0.001)$ (data available on request).

\section{DISCUSSION}

Black and minority ethnic (BME) MSM accounted for just over one in 10 MSM diagnosed with HIV in E\&W between 1997 and 2002. Where probable country of infection was known, more than half had probably acquired their infection within the United Kingdom. In 2002, in E\&W, a significantly higher proportion of BME MSM were living with diagnosed HIV infection than white MSM. Prevalence of undiagnosed HIV was high among Caribbean born MSM.

\section{Methodological issues}

Strengths of this analysis include triangulation of data from three national surveillance sources. In addition, results are based on laboratory confirmed HIV diagnoses. There are, however, some limitations. The number of BME men infected through sex between men may be underestimated because of misclassification of ethnicity and sexual orientation at the clinic level. Cultural taboos may inhibit disclosure of sex with another man and those having sex with both men and women may not perceive themselves to be bisexual, reporting heterosexual exposure only.$^{18}$ Without qualitative investigation it is impossible to quantify the extent of this misclassification.

In these analyses completeness of ethnicity data was high with over $90 \%$ of new diagnoses and SOPHID patients having ethnicity recorded. Nearly half the newly diagnosed BME MSM and those accessing services were classified as other/ mixed ethnicity, which in itself is not very informative unless combined with country of birth data; collection of such a broad ethnic category needs to be reviewed. Data on country of infection and birth for newly diagnosed BME MSM were incomplete and care should be taken when interpreting these results. It is difficult to ascertain how this incompleteness would bias results: for probable country of infection it is likely that where country of infection was uncertain it was not assigned.

Undiagnosed prevalence of HIV among BME MSM is based on a population from 14 GUM clinics, which may not fully represent BME MSM attending all 228 GUM clinics in E\&W. A further consideration is that clinic attendees will not be fully representative of all BME MSM as those attending GUM clinics are generally at higher risk of acquiring HIV. The UAPMP survey collects information on region of birth rather than ethnicity, which in some instances may not be a good proxy for ethnicity, particularly for MSM born in Africa and South America. ${ }^{19}$ The data presented in this paper indicate that nearly a third of newly diagnosed BME MSM were born in the United Kingdom. Using world region of birth as a proxy for ethnicity would not capture this information. Finally, it is important to note that although $52 \%$ of all MSM accepted an HIV test we could not ascertain how many men were offered a test. This data item has been introduced into the 2003 UAPMP GUM survey.

\section{Diagnosed HIV among BME MSM}

Between 1997 and 2002, BME MSM accounted for just over one in 10 new HIV diagnoses among MSM in E\&W. More than half had probably acquired HIV in the United Kingdom. BME MSM were younger at diagnosis than white MSM, suggesting infection with HIV at an earlier age or earlier uptake of HIV testing. They were not diagnosed any later during the course of infection as evidenced by AIDS diagnosis within 3 months of HIV diagnosis. Rising diagnoses combined with improved survival have led to increasing numbers of BME MSM attending HIV treatment and care services, 
with over one in 10 MSM attending services in 2002 being BME. Most lived in London. In E\&W in 2002, a significantly higher proportion of BME MSM were living with diagnosed HIV than white MSM. According to our estimates there was a twofold difference. The proportion of BME MSM attending NHS HIV treatment and care services receiving triple therapy (with a CD4 count of less than 200 cells $\times 10^{6} / 1$ ) did not differ significantly from white MSM. This is in stark contrast to the United States, where marked inequalities in HIV treatment and care exist between black people, Latinos and white people. ${ }^{20}{ }^{21}$ These differences may be explained by healthcare provision. Black people and Latinos in the United States are at economic disadvantage and are more likely to receive HIV care through publicly funded Medicaid rather than privately funded services. In the United Kingdom, HIV treatment and care services are typically provided free at the point of access to diagnosed patients, through the NHS.

\section{Undiagnosed HIV among BME MSM}

The prevalence of undiagnosed HIV among Caribbean born MSM exceeded that for other BME groups between 1997 and 2002. While there is no clear explanation for this differential, several factors could be contributing: high rates of bacterial STIs among black Caribbeans may be facilitating transmission of HIV ${ }^{22}{ }^{23}$; compared with white MSM, black MSM may be more likely to engage in unprotected anal intercourse with an HIV infected partner or with a partner of unknown HIV statu $^{24}$; there may be differential immigration to the United Kingdom of HIV infected MSM from the Caribbean, driven by local stigma and discrimination. ${ }^{25}{ }^{26}$ This wide disparity between black Caribbean MSM and other ethnicities merits further investigation. Other UAPMP results indicate that more than half the MSM who could have been diagnosed left the GUM clinic unaware of their infection, irrespective of where they were born. Conversely, between 1997-2002, VCT uptake increased significantly among MSM from all regions of birth, which may reflect promotion of HIV testing among clinic attendees, and is encouraging.

\section{Comparisons with other studies}

There are limited data with which to compare findings. Research into HIV testing and sexual risk behaviours among ethnically diverse MSM in a community based study in England in 2001 found that self reported HIV prevalence was 5.9\% (50/853) among BME MSM overall ("black," "Asian," and "all others" combined) compared to 5.3\% (655/12 462) among white MSM ("white British" and "white other" combined) $(p=0.4) .{ }^{24}$ The lack of a statistically significant difference in self reported HIV prevalence between BME and white MSM in this study is at odds with our own prevalence estimates based on laboratory confirmed HIV diagnoses. In 2002, we found a significant twofold difference in the estimated proportion of BME and white MSM living with diagnosed HIV in E\&W. However, the authors of the community based study did find that self reported HIV prevalence was higher among black MSM (11.3\% (35/309)) than among Asian and "all other" men (1.5\% (5/327) and $4.6 \%$ (10/217) respectively) $(\mathrm{p}<0.001)$. They recommended that HIV prevention programmes should prioritise black MSM. ${ }^{24}$ Our data would support this recommendation, with an elevated prevalence of undiagnosed HIV among black Caribbean MSM compared with other BME groups.

In the United States substantially higher levels of incident HIV infection have been reported among black MSM than white MSM, a disparity that is not explained by higher rates of unprotected anal and oral sex. ${ }^{97}$ Similarly, HIV prevalence is much higher among black MSM, reaching 32\% among those aged 23-29 years in metropolitan areas. ${ }^{9}$ Fortunately, HIV has not reached these levels among BME MSM in E\&W but undiagnosed HIV prevalence was high among Caribbean born MSM between 1997 and 2002 and this situation needs to be monitored. HIV prevalence ranging from $5 \%$ to $15 \%$ has been found among MSM in the Caribbean. ${ }^{25}$

\section{Implications of this study}

Our findings have important implications for HIV surveillance and health promotion in E\&W. Surveillance of HIV among BME MSM could be improved if data on ethnicity, country of birth and infection for new HIV diagnoses were complete. Reporting completeness, particularly for clinician HIV reports, needs to be addressed. The evidence of acquisition of HIV by BME MSM in E\&W makes them an emerging target group for health promotion. The elevated undiagnosed HIV prevalence in Caribbean born MSM is of concern, highlighting a need for black Caribbean MSM to be prioritised when planning health promotion initiatives. HIV rates among BME MSM in E\&W have not reached the level observed in the United States. None the less, high rates of bacterial STIs and sexual risk behaviours among black minority ethnic groups in E\&W compounded by racism and cultural taboos surrounding sexual orientation demand that we remain alert to the sexual health needs and evolving epidemiology of HIV among BME MSM in E\&W. Further research among BME MSM may provide an evidence base for those planning appropriate and culturally specific sexual health services.

\section{ACKNOWLEDGEMENTS}

We gratefully acknowledge the contribution of the many people who report to, and support, the national HIV surveillance systems, as well as the Health Protection Agency Sexually Transmitted and Bloodborne Viruses Laboratory for their work on the UAPMP. We also extend our gratitude to Dr Catherine Mercer (Natsal) and Dr Linda Lazarus (Department of Health), and to Kathleen Baster (Health Protection Agency) and André Charlett (Health Protection Agency) for statistical advice.

The Department of Health provides funding for the SOPHID and UAPMP surveys.

\section{CONTRIBUTORS}

SD conceived the idea for the paper with significant input from JE; $\mathrm{SD}, \mathrm{BR}$, and $\mathrm{AB}$ analysed the new diagnoses, SOPHID and UAPMP GUM surveys respectively, with support from KS and BE (new diagnoses and SOPHID), and OG (UAPMP); all authors were involved in interpretation of the results and drafting of the paper; SD undertook the main writing of the paper with JE and KF making a significant contribution. SD is currently registered for a PhD at City University London.

\section{Authors' affiliations}

S Dougan, B Rice, A E Brown, K Sinka, B G Evans, O N Gill, K A Fenton, Department of HIV and Sexually Transmitted Infections, Communicable Disease Surveillance Centre, Health Protection Agency Centre for Infections, 61 Colindale Avenue, London NW9 5EQ, UK

$S$ Dougan, J Elford, City University London, Institute of Health Sciences, St Bartholomew School of Nursing and Midwifery, UK

\section{REFERENCES}

1 Health Protection Agency, SCIEH, ISD, National Public Health Service for Wales, CDSC Northern Ireland and the UASSG. Renewing the focus. HIV and other sexually transmitted infections in the United Kingdom in 2002. London: Health Protection Agency, November 2003, (www.hpa.org.uk/infections/ topics_az/hiv_and_sti/publications/annual2003/annual2003.pdf).

2 Sinka K, Mortimer J, Evans B, et al. Impact of the HIV epidemic in subSaharan Africa on the pattern of HIV in the UK. AIDS 2003;17:1683-90.

3 Johnson AM, Mercer CH, Erens B, et al. Sexual behaviour in Britain: partnerships, practices, and HIV risk behaviours. Lancet 2001;358:1835-42. 4 Mercer CH, Fenton KA, Copas AJ, et al. Increasing prevalence of male homosexual partnerships and practices in Britain 1990-2000: Evidence from national probability surveys. AIDS 2004;18:1453-8.

5 Office for National Statistics. Census 2001. (www.statisitics.gov.uk/ downloads/census2001/National_report_EW_Part1_section2.pdf, accessed February 2004). 
6 Fenton KA. Strategies for improving sexual health in ethnic minorities. Curr Opin Infect Dis 2001;14:63-9.

7 Hughes G, Catchpole M, Rogers PA, et al. Comparison of risk factors for fou sexually transmitted infections: results from a study of attenders at three genitourinary medicine clinics in England. Sex Transm Infect 2000;76:262-7.

8 Fenton K, Cadette M, Boakye P, et al. Good practice guidelines for HIV health promotion with black gay and bisexual men. London: Published on behalf of the CHAPS Partnership, September, 2002.

9 Centers for Disease Control and Prevention. HIV incidence among young men who have sex with men-seven US cities, 1994-2000. MMWR Morb Mortal Wkly Rep 2001;50:440-1.

10 PHLS Communicable Disease Surveillance Centre, ICH (London), SCIEH. HIV \& AIDS in the UK. An epidemiological review, 2000.London, 2001.

11 McHenry A, MacDonald N, Sinka K, et al. National assessment of prevalent diagnosed HIV infections. Commun Dis Public Health 2000;3:277-81.

12 Unlinked Anonymous HIV Surveys Steering Group. Prevalence of HIV in the United Kingdom, Data to the end of 1998. London: Department of Health, Public Health Laboratory Service, Institute of Child Health (London), Scottish Centre for Infection and Environmental Health, 1999.

13 Mortimer JY, Salathiel JA. "Soundex" codes of surnames provide confidentiality and accuracy in a national HIV database. Commun Dis Rep 1995; 15:R183-6

14 Heptonstall J, Gill ON. The legal and ethical basis for unlinked anonymous testing. Commun Dis Rep 1989:48:3-6.

15 Medical Research Council. Guidance on "collections of human tissue and biological samples for use in human research. London: MRC, April, 2001

16 Department of Health. Clinical Ethics and Human Tissue Branch. The use of human organs and tissue-an interim statement. London: DoH, April, 2003.
17 PHLS Communicable Disease Surveillance Centre. Quarterly Communicable Disease Review October to December 2001: Caldicott, Confidentiality and the Patient Information Advisory Group. J Public Health Med 2002;24:138-44.

18 Elam G, Fenton K, Johnson A, et al. Exploring ethnicity and sexual health. London: Social and Community Planning Research, 1999:1-116.

19 Dougan S, Elford J, Sinka K, et al. Men who have sex with men who are born abroad and diagnosed with HIV in England and Wales: an epidemiological perspective. Int J STD AIDS, (in press)..

20 Shapiro MF, Morton SC, McCaffrey DF, et al. Variations in the Care of HIVinfected adults in the United States. JAMA 1999:281:2305-15.

21 Halkitis PN, Parsons JT, Wolitski RJ, et al. Characteristics of HIV antiretroviral treatments, access and adherence in an ethnically diverse sample of men who have sex with men. AIDS Care 2003;15:89-102

22 Low N, Sterne JAC, Barlow D. Inequalities in rates of gonorrhoea and chlamydia between black ethnic groups in southeast London: cross sectional study. Sex Transm Infect 2001;77:15-20.

23 Radcliffe KW, Ahmad S, Gilleran G, et al. Demographic and behavioural profile of adults infected with chlamydia: a case-control study. Sex Transm Infect 2001;77:265-70.

24 Hickson F, Reid D, Weatherburn $P$, et al. HIV, sexual risk and ethnicity among men in England who have sex with men. Sex Transm Infect 2004;88:443-50.

25 Cáceres CF. HIV among gay and other men who have sex with men in Latin America and the Caribbean: a hidden epidemic? AIDS 2002;16/suppl 3):S23-S33.

26 Keogh P, Dodds C, Henderson L. Migrant gay men: redefining community, restoring identity, Sigma Research, February, 2004.

27 Malebranche DJ. Black men who have sex with men and the HIV epidemic: next steps for public health. Am J Public Health 2003;93:862-5. 